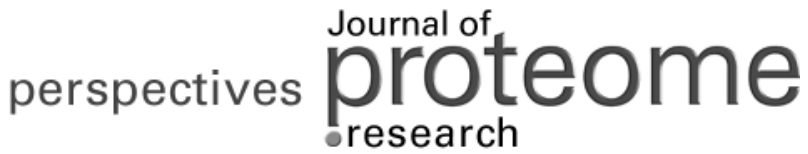

\section{Perspective: A Program to Improve Protein Biomarker Discovery for Cancer $^{\dagger}$}

\author{
Ruedi Aebersold, Leigh Anderson, Richard Caprioli, Brian Druker, Leland Hartwell,* and \\ Richard Smith
}

\author{
Fred Hutchinson Cancer Research Center, 1100 Fairview Avenue, North, PO Box 19024, \\ Seattle, Washington 98109-1024 \\ Received February 11, 2005
}

\begin{abstract}
Biomarkers for cancer risk, early detection, prognosis, and therapeutic response promise to revolutionize cancer management. Protein biomarkers offer tremendous potential in this regard due to their great diversity and intimate involvement in physiology. An effective program to discover protein biomarkers using existing technology will require team science, an integrated informatics platform, identification and quantitation of candidate biomarkers in disease tissue, mouse models of disease, standardized reagents for analyzing candidate biomarkers in bodily fluids, and implementation of automation. Technology improvements for better fractionation of the proteome, selection of specific biomarkers from complex mixtures, and multiplexed assay of biomarkers would greatly enhance progress.
\end{abstract}

Keywords: biomarker $\bullet$ cancer $\bullet$ discovery $\bullet$ research platform • informatics $\bullet$ reagents

\section{Introduction}

Biomarkers are becoming increasingly important in cancer, and it is clear that cancer patients would benefit enormously by a greater availability of such effective molecular indicators that can be monitored noninvasively from readily accessible bodily fluids. Biomarkers are defined as endogenous or injected molecules whose presence or metabolism correlates with important disease related physiological processes and/or disease outcomes. They should be identified or defined molecular entities to facilitate comparison across laboratories and technology platforms. In recent years, progress has been strong in the discovery and implementation of nucleic acid biomarkers, but the identification of protein biomarkers is as yet less advanced. The purpose of this article is to discuss and help focus the means by which we may accelerate protein biomarker discovery.

\section{Biomarkers in Cancer Therapy}

The molecular analysis of cancer presents a genetic landscape that is orthogonal to the standard organ-specific view. Within an organ several genetic pathways may lead to cancer, and the same sets of altered genes appear across organs. This situation is leading to an increasing emphasis on the genetic characterization of cancer as biomarkers for prognosis and therapeutic intervention. With this comes the concomitant advantage of measuring proteins, the products of these genes. Since functional proteins may change significantly on

\footnotetext{
† Part of the Biomarkers special issue.

* To whom correspondence should be addressed. Fred Hutchinson Cancer Research Center, Mail Stop D1-060, 1100 Fairview Avenue, North, PO Box 19024, Seattle, WA 98109-1024. Tel: (206) 667-5670. Fax: (206) 667-5268 E-mail: lhartwel@fhcrc.org.
}

a temporal and geographical basis, protein biomarkers promise to have a significant impact in cancer detection and therapy.

More accurate diagnosis of the hundreds of different types of cancer will permit more effective choice of therapy and will enhance clinical trials. Indeed, as therapies become more targeted to specific signal transduction and metabolic pathways, it is becoming of paramount importance to document the existence of those pathways in the target cancers. Consider some specific genomic examples. Targeting of breast cancers with HERCEPTIN (trastuzumab) is not indicated if the patient's tumor does not overexpress Her-2/neu. ${ }^{1,2}$ Similarly, GLEEVEC (imatinib) is most effective against cancers that carry the bcrAbl translocation. ${ }^{3}$ Genetic alterations or transcript array profiles allow the stratification of many organ-specific cancers (e.g., breast, leukemia, lymphoma, sarcoma) into different subtypes that have distinctive therapeutic outcomes. For example, Myc gene amplification status predicts outcome for childhood neuroblastoma. ${ }^{4}$ The quantity of Bcr-Abl transcript predicts risk of disease recurrence in chronic myelogenous leukemia (CML) long before clinical symptoms recur. ${ }^{5}$ As these examples suggest, it will be important to develop informative biomarkers for virtually all cancer subtypes.

Real-time markers of the physiological state of the patient, whether markers of disease process or those indicating efficacy of treatment will bring enormous benefit to patient care. For example, this would greatly improve individualized dosing and agent selection. Indeed, some chemotherapeutic agents are currently individualized by adjusting the dose according to the patient's individual metabolic characteristics. Moreover, a series of agents could be tested on the same patient in a matter of weeks to determine empirically the most effective therapy. 


\section{Biomarkers in Early Detection}

Short of prevention, improved diagnostics to detect cancer at an early stage, when it is curable with contemporary methods, would provide the greatest benefit for cancer patients. For most cancers, five- and even ten-year survival often approaches $90 \%$ for cancer detected at an early stage, while it may drop to $10 \%$ or less for cancer detected at a late stage. ${ }^{6}$ It is well established that screening to detect cancer earlier saves lives. For example, the PAP smear strongly reduces mortality through early detection of pre-neoplastic cervical cancer lesions, ${ }^{7}$ as does colonoscopy for colon cancer. ${ }^{8,9}$ Furthermore, both tests have been widely employed despite their significant inconvenience, unpleasantness, cost, and requirement for clinical expertise. These successful protocols have created a receptive social environment that will encourage the rapid application of new tests. What is needed, however, are affordable and effective diagnostic tests for more types of cancer.

The risk of cancer recurrence is high in those patients who have previously had cancer, even for those who have been in remission for five or more years. Cancer survivors constitute a high-risk group that would benefit from improved tests for early detection of disease recurrence if effective therapy is available. Monitoring CML patients during GLEEVEC therapy and in the post-transplant setting for the persistence of the Bcr-Abl transcript is already an effective technique for detecting disease recurrence at an early stage. ${ }^{3,10}$

\section{Biomarkers in Risk Assessment}

If we could segment the population into smaller groups at increased risk for specific cancers, then screening individuals for early cancer detection will be more cost-effective and produce fewer unnecessary interventions. Molecular biomarkers that predict risk are essential. Success in identifying individuals at increased risk has been achieved for many cancers through epidemiological studies that identify strong environmental or behavioral risk factors and by genetic studies that identify mutations underlying rare inherited cancer syndromes. However, the use of biomarkers in the assessment of risk for sporadic disease remains largely unexplored.

Epidemiologic studies indicate that lifestyle, diet, and environmental exposures significantly affect the risk for sporadic disease, but few advances have been made in identifying biomarkers that reflect the stable, cumulative molecular changes associated with disease or mediate this risk. While stochastic genetic alterations occur infrequently and are difficult to detect, there is increasing interest in more common, stable genetic, and epigenetic changes in histologically normal or premalignant tissue, reflective of deleterious exposure and associated with increased risk for malignant progression. For example, DNA mutation, methylation, and ploidy changes are highly correlated with an increased risk for cancer of the esophagus. ${ }^{11,12}$ Similarly, the loss of imprinting of insulin-like growth factor 2 (IGF2) in peripheral blood lymphocytes in subjects at risk for colorectal cancer is a risk marker. ${ }^{13,14}$

Many familial cancer-prone syndromes are due to defects in DNA repair. A study by Scott and Roberts revealed that approximately $40 \%$ of breast cancer patients, prior to treatment, exhibit a defect in DNA double strand break repair in their white blood cells. ${ }^{15}$ Therefore, biomarkers related to DNA repair capacity might be effective for risk stratification. For example, 8-oxoguanine DNA $N$-glycosylase (OGG) activity in peripheral blood monocytes is associated with risk of lung cancer. ${ }^{16}$

\section{Noninvasive Monitoring of Biomarkers}

Individuals at risk for cancer or with cancer would benefit enormously by better methods for determining cancer risk, detecting and localizing cancer at its earliest stage, profiling for therapeutic decision making, and monitoring response to therapy in real time. For some of these applications, we will not know whether a tumor exists or, if so, its anatomical site. Thus, there is a need for biomarkers that can be monitored noninvasively in readily available bodily fluids.

Tumors "leak" DNA and proteins into circulation. Tumors also induce dramatic alterations of surrounding stroma (e.g., alterations in basement membranes, angiogenesis, and lymphogenesis) and release proteases that digest normal tissue and plasma proteins. It is therefore reasonable to expect many biomarkers to be present in blood and other fluids. Indeed, several individual plasma proteins (i.e., prostate-specific antigen [PSA], cancer antigen 125 [CA125], carcinoembryonic antigen [CEA], and alpha fetoprotein [AFP] antigen) are in clinical use as markers of the presence of a tumor, response to therapy or of tumor recurrence. ${ }^{17,18}$

Progress is being made in detecting mutated or methylated tumor DNA in accessible bodily fluids including blood, urine, sputum, and stools. ${ }^{19,20}$ Academic and commercial entities are currently developing highly sensitive and specific panels of biomarkers for different cancer sites. DNA biomarkers found in bodily fluids may change with tumor progression and correlate with aggressiveness and therapeutic response.

In addition to DNA- and RNA-based diagnostics, other molecules offer diagnostic potential; many new contrast agents for molecularly targeted imaging are under development. The technology for discovering and developing nucleic acid biomarkers and new imaging agents is robust and is being actively pursued in academic and commercial laboratories.

\section{Proteins as Biomarkers}

The greatest potential for enabling biomarkers for cancer lies in improving the technology for protein biomarker discovery. Moreover, there is also interest in the potential of using other molecules, such as small molecules (metabolomics) and carbohydrates (glycomics) as biomarker diagnostics, but the technologies for discovering these biomarkers are still nascent.

Proteins are more diverse than DNA or RNA and therefore carry more information than nucleic acids, since alternative splicing and more than 100 unique post-translational modifications result in tens (and possibly hundreds) of species of protein from each gene. Moreover, many physiologic changes are mediated post-transcriptionally and will not be revealed at the nucleic acid level. Proteins are also more dynamic and reflective of cellular physiology. For example, the occurrence of a single double-strand DNA break in a cell is rapidly amplified into a cascade of protein phosphorylation.

\section{Current State of Protein Biomarker Technology}

Technology limits our capacity to interrogate the proteome of complex protein mixtures (e.g., blood or tissue) for biomarker discovery. It has been estimated that blood contains more than 100000 different protein forms with abundances that span 1012 orders of magnitude. ${ }^{21}$ Mass spectrometry (MS), the leading tool in proteomics, currently has a limited capacity to identify and quantitate proteins in complex mixtures. ${ }^{22}$ Proteins are often digested to 20 and 50 peptides, further increasing the complexity of species in a mixture. Mass spectrometers that 


\section{perspectives}

use electrospray ionization provide high resolving power for small mass differences, and tandem instruments (MS/MS) can identify a peptide's protein of origin by examining the peptide pattern following induced fragmentation. While quantitation of proteins is not straightforward, it can be achieved using internal standards. This technology allows researchers to identify hundreds of the most abundant proteins from a complex mixture. Other mass spectrometric techniques, such as matrix-assisted laser desorption ionization/time-of-flight (MALDI-TOF) MS, can display hundreds of the most abundant intact proteins in a mixture, although identification of the proteins is not possible without applying additional laborious technologies.

Most of the known biomarkers in blood occur at very low abundance and would not be revealed by either of these techniques. These facts lead to several conclusions. First, simple profiling of bodily fluids that contain complex mixtures of proteins that span huge dynamic ranges is unlikely to be very effective with present technologies. Therefore, candidate biomarkers will need to be identified prior to an attempt to correlate these biomarkers in bodily fluids with disease status. A corollary of this conclusion is that the subsequent analysis of these candidates in bodily fluids will likely require reagents specific to each candidate to detect and quantitate them at lowabundance.

\section{Identifying Candidate Protein Biomarkers}

Any proteins that are differentially expressed in cancer tissue when compared to normal tissue, or any proteins that are known to be involved in the cancer process, are good sources of candidate biomarkers for cancer. One of the standard methods to identify candidate biomarkers from the first category is to compare transcription profiles from disease vs normal tissue to identify differentially expressed transcripts. Another approach that has received attention is to identify genes amplified in cancer tissues. While these are fruitful approaches, they will not detect the vast majority of protein biomarkers-those that arise by post-transcriptional mechanisms. Direct identification of proteins that are differentially present in cancer tissue due to changes in translation, degradation, or post-translational modification is well within the capacity of MS or even 2D-gel analysis followed by LC-MS. Given the limitations of both methods, it is reasonable to expect to be able to identify hundreds of candidates from any particular fraction of disease tissue. Although these methods will be biased toward the abundant proteins, it is the abundant proteins that are more likely to be present in significant quantities in blood. One of the several approaches that may be useful is the identification of specific protein markers of tumor viability in tumor tissue itself. These markers, or protease fragments of these, could then be specifically targeted for analysis in body fluids. This may lead to "high quality" biomarkers directly associated with the disease tissue.

For the analysis of body fluids, the question is which fraction(s) to examine. Here, we are in unknown territory, since we do not know which fraction of cellular proteins is more likely to be enriched for biomarkers that can ultimately be identified in blood. Some fractions worth examining are cell surface proteins, secreted proteins, phosphorylated or glycosylated proteins, products of proteolytic digestion, etc. Analysis of any one of these fractions could be done either following tumor cell enrichment, which has the advantage of amplifying the signals over normal tissue, or in unfractionated tumor tissue, which offers the advantage of preserving stromal cell proteins that may also be valuable markers of disease. No single laboratory can hope to examine all these sources comprehensively, and this consideration suggests that teams will need to collaborate on identification of biomarkers for even a single cancer type.

In our search for biomarker candidates, we should not overlook the wealth of accumulated knowledge about cancer and cell biology. During the last forty years, we have achieved an impressive understanding of the molecular fundamentals of cancer. We now understand that cancer arises from a single cell as a result of successive genetic changes that alter a number of cellular processes, including growth control, senescence, apoptosis, angiogenesis, and metastasis. ${ }^{23}$ Additionally, many cancers appear to have activated inflammation and woundhealing genetic expression programs. ${ }^{24}$ These changes are driven by abnormal methylation or mutation. While the proteins that function in each of these cellular circuits are often thought of as drug targets, they also represent strong candidates for biomarkers.

Bioinformatics approaches can easily generate a list of hundreds of proteins associated with each of these processes. Examining the bodily fluids of cancer patients for all of these proteins will require reagents in the form of antibodies, aptamers, and/or isotopically labeled peptides. No single laboratory can be expected to produce the reagents for even a single process, and even if the reagents were made available, no single laboratory could examine the proteins in the blood of cancer patients for more than one process. These considerations lead to a need for teams of laboratories concentrating on a single cancer site and for a centralized source of standardized reagents with known performance characteristics.

In addition to the large number of protein categories that must be analyzed to discover biomarker candidates, there is also the issue of choice of bodily fluid to examine as the ultimate source of diagnostic information. Investigators are currently examining plasma, serum, urine, sputum, and other materials, and there is no consensus as to the best site for even one specific cancer. This fact increases the dimensionality of the problem by requiring that biomarker discovery be pursued in as many different bodily fluids as possible, further reinforcing the need for team science.

\section{Value of Mouse Models of Cancer}

It is highly unlikely that any set of human samples, whether tissue or fluids, will yield a set of biomarkers diagnostic of cancer at the desired $100 \%$ sensitivity and specificity. This is because humans are genetically and environmentally diverse, many clinical subtypes of tumors exist at any organ site, samples are collected from patients by different caregivers at different treatment centers under a variety of conditions and that there are ethical limits on the acquisition of samples for discovery. To assess technology performance, one must minimize this heterogeneity as much as possible. Consequently, ongoing technology assessment using pooled samples of bodily fluids from inbred mice containing pre-engineered cancerinducing mutations across laboratories will assist in identifying cancer biomarkers and minimize confounding factors. Such samples will help to control for both germ-line and somatic heterogeneity (e.g., the clinical heterogeneity of tumors). Moreover, all mice representative of a particular cancer site could be bred at a single location, and all samples could be 
TECHNOLOGY APPLICATION
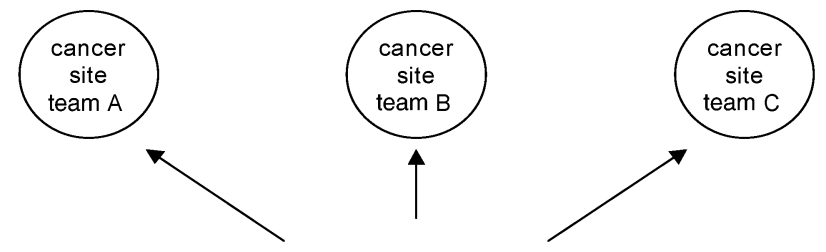

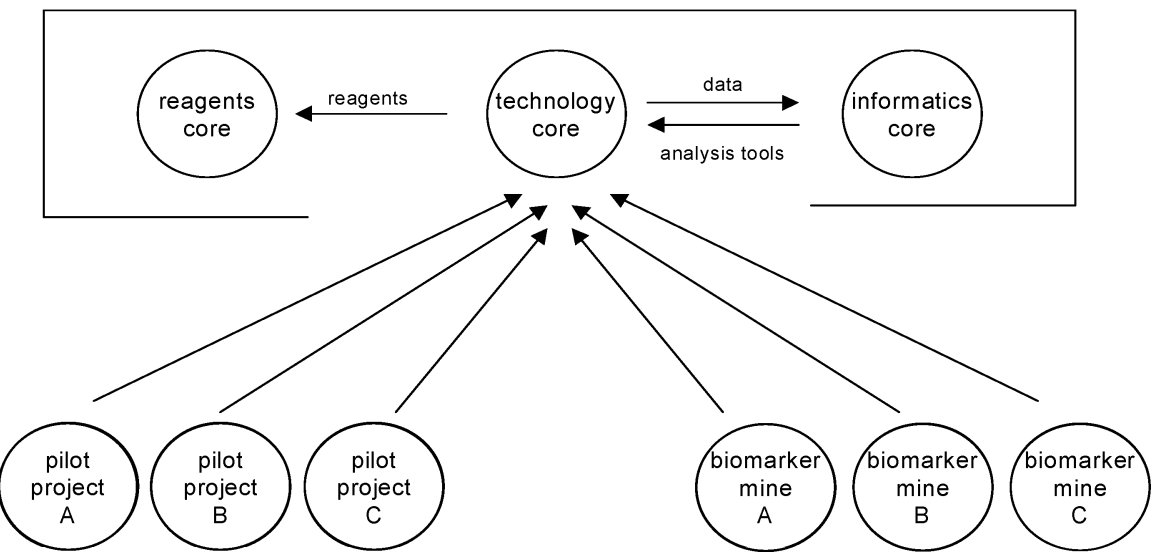

Figure 1. Fully integrated clinical biomarker discovery technology program centers around Core resources for technology assessment, reagents, and informatics. New technologies will be evaluated through pilot projects and "biomarker mines" comprised of individual investigators and smaller research teams. Upon standardization by the Cores, new proteomic technologies can be tested in the clinical setting by Cancer Site Teams dedicated to biomarker discovery at a particular cancer site.

collected and processed by the same individuals in a single laboratory at uniform stages of development, ages, and time of day.

It will be crucial to determine whether the technology platforms can deliver cancer detection biomarkers with high specificity and sensitivity under these ideal conditions. If investigators succeed under these ideal conditions, then it will become clear that poorer performance with human samples is likely due to one of the above sources of heterogeneity. It will then be appropriate either to invest resources in determining systematically the sources of heterogeneity in human samples or to sample a larger cohort of humans to increase the statistical power to detect biomarkers despite heterogeneity. Any test for cancer detection in humans will need extensive standardization and characterization for sensitivity and specificity.

Biomarker discovery in mouse models of human cancer may make it feasible to identify mouse orthologs of human biomarkers. This outcome is possible since mouse cells pass through many of the same physiologic processes as human cells (e.g., apoptosis, angiogenesis, metastasis). For these reasons, and to calibrate technology performance, the use of mouse models along with human samples for biomarker discovery is a necessity.

\section{Organization of a Program in Protein Biomarker Discovery}

Figure 1 depicts components of a program that emerges from the considerations given above. The proposed initiative is structured into three integrated core components: Informatics, Reagents, and Technology Assessment. In addition to its development function, each core component would support, through services and resources, various satellite projects organized around cancer sites, biomarker mines, and pilot projects. These components do not need to be localized at a single physical site; with the aid of information technology, they can be "virtual" sites.

\section{Cancer Site Teams}

The Cancer Site Teams shown in Figure 1 represent groups of investigators dedicated to biomarker discovery at a particular cancer site, e.g., breast, lung, prostate, and colon. Each team would have clinicians with access to human tissue at that site, scientists working on mouse models of the particular cancer, and other researchers who are skilled in protein fractionation and enrichment techniques, antibody preparation, mass spectrometry, and informatics.

\section{Biomarker Mines}

The Biomarker Mine components would comprise single investigators or small groups dedicated to optimizing the methods for discovery in a particular category of biomarkers, such as cell surface or secreted proteins. Optimization of procedures for tissue workup, bioinformatics, and the biochemical fractionations and analysis for a specific category of biomarkers requires special attention that is unlikely to occur in the Cancer Site Teams who are more committed to discovering biomarkers for a particular cancer site. The Biomarker Mine components would instead develop analytical methods to identify candidate biomarkers in a specific mine, identify and rank prospective candidates, develop reagents for their enrichment and detection, work collaboratively with one or more Cancer Site teams and the Technology Assessment Core to mine for cancer-specific biomarkers, deposit data into the Informatics Core, and share data and reagents with other components.

\section{Informatics Platform}

The Informatics Core will develop tools that enable laboratories to communicate efficiently and to compare data. This 


\section{perspectives}

core would develop a standardized data format to facilitate cross-platform comparisons. It would also provide an opensource suite of analysis tools compatible with this standard data format to facilitate standardization of analysis across laboratories and allow meaningful comparisons of results. Additionally, a central database for storing the data of the programs would be housed in the Informatics Core. Finally, the Informatics Core will assemble and curate data sources for candidate biomarkers.

\section{Reagents Core}

The Reagents Core would organize tools for biomarker discovery, maintaining a virtual database of reagents, their characteristics, and their performance data, along with reagent request forms. Reagents as defined here include mice, mouse and human tissues and plasma, antibodies for candidate enrichment, standard batches of bodily fluids, standard protein and/or peptide mixtures for spiking fluids prior to analysis, and other standard reagents developed or discovered by investigators. Reagents, along with data on their performance and quality, will be acquired and dispersed quickly to other core facilities and satellites.

One of the most frequently identified needs in the biomarker discovery field is for high quality tissues from cancer patients and controls. It is unclear at present how best to collect, prepare, and store tissue or bodily fluids. For this reason, tissue collections should probably be coordinated at the inception of a project and occur prospectively. The greatest confounder of effective biomarker discovery is the false positives generated because the control tissue is not matched sufficiently to the disease tissue. There are several possible sources of valid control tissue, including "normal" tissue from the same organ and individual as the cancer, normal tissue from other healthy individuals (including identical twins where possible) and tissue from individuals with disease of the same organ but of a different nature (e.g., inflammation). Ideally, each should be compared with disease tissue. Initial evaluation of biomarkers will require hundreds of clinically annotated tissue and blood samples. To evaluate early detection capability, plasma must be collected from early-stage patients (together with stored tissue) as well as from pre-symptomatic individuals who are later diagnosed with cancer. To evaluate clinical response, plasma obtained from well-controlled clinical trials with clinical outcomes is essential.

For protein biomarker discovery, it is essential to have access to many antibodies for detection of candidates that are likely to be present at low concentrations in the blood. One could easily create a list of more than a thousand proteins known to be involved in cancer-related processes, each of which is a potential candidate biomarker. Moreover, as candidate proteins are discovered by proteomic methods that distinguish disease from normal tissue, additional antibodies will be needed for these candidates. Several thousand antibodies will likely be required, as pairs of antibodies for enzyme-linked immunosorbent assays (ELISA) or other tests, such as proximity-based detection. Many of the antibodies developed for a particular cancer site will be reused at other cancer sites, thereby promoting efficient use of resources.

In addition to antibody-based methods, other approaches, such as VICAT, are available to identify and quantitate a candidate peptide at low concentration in complex mixtures such as blood. ${ }^{25}$ Anti-peptide antibodies will be useful for
Aebersold et al.

enrichment in the SISCAPA approach, ${ }^{26}$ and appropriate isotopically labeled peptide reagents will be needed.

\section{Incorporating Technology Improvements}

Although it is possible to improve the discovery of protein biomarkers, the performance of technologies for protein separation and identification remains the major limitation to translate biomarker discovery into therapies and diagnostics. Technology improvements are likely to occur over the next few years, and these advances should be rapidly incorporated into the discovery process. Technology improvement should be considered in mass spectrometry, protein arrays, protein fractionation, protein detection, protein quantitation, and other appropriate methodologies.

To support such efforts, the Technology Assessment Core will evaluate mature, commercialized technologies central to biomarker discovery to provide laboratories with the best possible techniques and protocols. Initially, this Core would use standard reference plasma to systematically compare existing technologies in each step of the biomarker discovery process. The best-performing technologies would then be integrated into an optimized platform against which new technologies (discovered via pilot projects and biomarker mines; see Figure 1) could be tested. The ability of the integrated and optimized platform to identify biomarkers would likely best be assessed using mouse models. This Core would also provide data to the Informatics Core for algorithm development and would deposit useful reagents (including reference plasma and mouse tissues) into the Reagents Core for dissemination. Finally, this Core would collaborate with Cancer Site components to implement optimized technologies to identify biomarkers in human samples. This Core might be a virtual core that requests performance criteria from investigators with new technology.

To encourage new technology development prior to commercialization a pilot project program might be useful. The pilot projects would be single-investigator projects designed to test a new technology, such as protein chips for biomarker discovery. Where appropriate, promising new technologies would be reproduced and tested against current standards by the Technology Assessment Core.

\section{Conclusion: A Demonstration Project}

This brief document is intended to express the enormous magnitude of the task needed to improve protein biomarker discovery. One might argue that we should wait for the technology to improve. However, this position is untenable for several reasons. First and foremost is the tremendous promise that protein biomarkers have for improving cancer patient outcomes. Second, technology improvement is often driven by a major initiative that strives for a goal slightly beyond reach. Third, vast improvements can often be made through standards, reagents, tools, and automation that do not in themselves constitute new technology. All of these principles were revealed by the Human Genome Project and are applicable here. However, given the magnitude of the project and the uncertainty surrounding biomarker performance, it would be reasonable to stage the implementation of these recommendations, beginning with one or two cancer sites and enlarging the activity if these are successful.

Acknowledgment. The ideas expressed here were gathered over the last two years in response to a request by Dr. 
Andy von Eschenbach asking L.H. to investigate how protein biomarker discovery could be improved. A number of meetings were organized by the NCI with the leadership of Anna Barker and Greg Downing. In addition to the input provided by the many scientists who participated in these meetings, we have also benefited from ongoing discussions with Sam Hanash, Amanda Paulovich, and Martin McIntosh.

\section{References}

(1) Baselga, J.; Gianni, L.; Geyer, C.; Perez, E. A.; Riva, A.; Jackisch, C. Future options with trastuzumab for primary systemic and adjuvant therapy. Semin. Oncol. 2004, 31, 51-57.

(2) Ross, J. S.; Fletcher, J. A.; Bloom, K. J.; Linette, G. P.; Stec, J.; Symmans, W. F.; Pusztai, L.; Hortobagyi, G. N. Targeted therapy in breast cancer: the HER-2/neu gene and protein Mol. Cell. Proteomics 2004, 3(4), 379-398, Apr.

(3) Druker, B. J. Imatinib as a paradigm of targeted therapies. Adv. Cancer Res. 2004, 91, 1-30.

(4) Schmidt, M. L.; et al. Biologic factors determine prognosis in infants with stage IV neuroblastoma: a prospective Children's Cancer Group Study. J. Clin. Oncol. 2000, 18, 1260-1268.

(5) Radich, J. P.; Gehly, G.; Gooley, T.; Bryant, E.; Clift, R. A.; Collins, S.; Edmands, S.; Kirk, J.; Lee, A.; Kessler, P.; Schoch, G.; Buckner, C. D.; Sullivan, K. M.; Appelbaum, F. R.; Thomas, E. D. Polymerase chain reaction detection of the BCR-ABL fusion transcript after allogeneic marrow transplantation for chronic myeloid leukemia: results and implications in 346 patients. Blood 1995, 85, $2632-2638$.

(6) Etzioni, R.; Urban, N.; Ramsey, S.; McIntosh, M.; Schwartz, S.; Reid, B.; Radich, J.; Anderson, G.; Hartwell, L. The case for early detection. Nat. Rev. Cancer 2003, 3, 243-252.

(7) Shingleton, H. M.; Patrick, J. L.; Johnston, W. W.; Smith, R. A. The current status of the Papanicolau smear Cancer 1995, 45(5), 305-320.

(8) Winawer, S. J.; St. John, D. J.; Bond, J. H.; Rozen, P.; Burt, R. W.; Waye, J. D.; Kronborg, O.; O'Brien, M. J.; Bishop, D. T.; Kurtz, R. C.; et al. Prevention of colorectal cancer: guidelines based on new data. WHO Collaborating Center for the Prevention of Colorectal Cancer. Bull. World Health Organ. 1995, 73, 7-10.

(9) Winawer, S. J. A quarter century of colorectal cancer screening: progress and prospects. J. Clin. Oncol. 2001, 19(18 Suppl), 6S12S, Sept 15

(10) Oehler, V. G.; Radich, J. P. Monitoring bcr-abl by polymerase chain reaction in the treatment of chronic myeloid leukemia Curr. Oncol. Rep. 2003, 5(5), 426-35, Sep.

(11) Zochbauer-Muller, S.; Lam, S.; Toyooka, S.; Virmani, A. K.; Toyooka, K. O.; Seidl, S.; Minna, J. D.; Gazdar, A. F. Aberrant methylation of multiple genes in the upper aerodigestive tract epithelium of heavy smokers. Int. J. Cancer 2003, 107, 612-616.

(12) Paulson T. G.; Reid, B. J. Focus on Barrett's esophagus and esophageal adenocarcinoma Cancer Cell. 2004, 6(1), 11-16.
(13) Jirtle, R. L. IGF2 loss of imprinting: a potential heritable risk factor for colorectal cancer. Gastroenterology 2004, 126, 1190-1193.

(14) Cui, H.; Cruz-Correa, M.; Giardiello, F. M.; Hutcheon, D. F.; Kafonek, D. R.; Brandenburg, S.; Wuy, Y.; He, X.; Powe, N. R.; Feinberg, A. P. Loss of IGF2 imprinting: a potential marker of colorectal cancer risk. Science 2003, 299(5613), 1753-1755.

(15) Scott, D.; Barber, J. B. P.; Spreadborough, A. R.; Burrill, W.; Roberts, S. A. Increased chromosomal radiosensitivity in breast cancer patients: a comparison of two assays. Int. J. Radiat. Biol. 1999, 75, 1-10.

(16) Paz-Elizur, T.; Krupsky, M.; Blumenstein, S.; Elinger, D.; Schechtman, E.; Livneh, Z. DNA repair activity for oxidative damage and risk of lung cancer. J. Natl. Cancer Inst. 2003, 95, 1312-1319.

(17) Bast, R. C., Jr.; Ravdin, P.; Hayes, D. F.; Bates, S.; Fritsche, H., Jr.; Jessup, J. M.; Kemeny, N.; Locker, G. Y.; Mennel, R. G.; Somerfield, M. R. 2000 update of recommendations for the use of tumor markers in breast and colorectal cancer: clinical practice guidelines of the American Society of Clinical Oncology. J. Clin. Oncol. 2001, 19, 1865-1878.

(18) Dhanasekaran, S. M.; Barrette, T. R.; Ghosh, D.; Shah, R.; Varambally, S.; Kurachi, K.; Pienta, K. J.; Rubin, M. A.; Chinnaiyan, A. M. Delineation of prognostic biomarkers in prostate cancer. Nature 2001, 412, 822-826.

(19) Muller, H. M.; Widschwendter, M. Methylated DNA as a possible screening marker for neoplastic disease in several body fluids Exp. Rev. Mol. Diagnost. 2003, 3(4), 443-458.

(20) Palmisano, W. A.; Divine, K. K.; Saccomanno, G.; Gilliland, F. D.; Baylin S. B.; Herman, J. G.; Belinsky, S. A. Predicting lung cancer by detecting aberrant promoter methylation in sputum. Cancer Res. 2000, 60(21), 5954-5958.

(21) Anderson, N. L.; Anderson, N. G. The human plasma proteome: history, character, and diagnostic prospects. [erratum appears in Mol. Cell Proteomics 2003, 2(1), 50, Jan] Mol. Cell. Proteomics 2002, 1(11), 845-867.

(22) Patterson, S. D.; Aebersold, R. H. Proteomics: the first decade and beyond. Nat. Genet. 2003, 33 Suppl, 311-323.

(23) Hanahan, D.; Weinburg,, R. A. The Hallmarks of Cancer Cells 2000, 100, 57-70.

(24) Chang, H. Y.; Sneddon, J. B.; Alizadeh, A. A.; Sood, R.; West, R. B.; Montgomery, K.; Chi, J. T.; van de Rijn, M.; Botstein, D.; Brown, P. O. Gene expression signature of fibroblast serum response predicts human cancer progression: similarities between tumors and wounds. PloS. Biol. 2004, 206-214.

(25) Bottari, P.; Aebersold, R.; Turecek, F.; Gelb, M. H. Design and synthesis of visible isotope-coded affinity tags for the absolute quantification of specific proteins in complex mistures. Bioconjugate Chem. 2004, 15(2), 380-388.

(26) Anderson, N. L.; Anderson, N. G.; Haines, L. R.; Hardie, D. B.; Olafson, R. W.; Pearson, T. W. Mass spectrometric quantitation of peptides and proteins using stable isotope standards and capture by anti-peptide antibodies (SISCAPA). J. Proteome Res. 2004, 3, 235-244.

PR050027N 